\title{
The effect of Kinesio Taping on spasticity: a randomized controlled double-blind pilot study in para-swimmers
}

Luca Puce, Ilaria Pallecchi, Lucio Marinelli, Maria May, Laura Mori, Piero Ruggeri, and Marco Bove

Puce, Marinelli, and Mori are with the Department of Neuroscience, Rehabilitation, Ophthalmology, Genetics, Maternal and Child Health, University of Genoa, Genoa, Italy.

Pallecchi is with the Physics Department, CNR-SPIN, Genoa, Italy.

Marinelli and Mori are also with the Department of Neuroscience, IRCCS Ospedale Policlinico San Martino, Genoa, Italy.

May is with the Department of Thoracic Surgery, Klinikum Bogenhausen, Munich, Germany.

Ruggeri and Bove are with the Department of Experimental Medicine, Section of Human Physiology, University of Genoa, Genoa, Italy.

Puce (lucalpuce@gmail.com) is corresponding author.

This article has been published in the Journal of Sport Rehabilitation on August 12, 2020 with

DOI: https://doi.org/10.1123/jsr.2019-0504 


\section{ABSTRACT}

Context: Kinesio Taping (KT) produces several clinical effects, including pain relief, edema absorption and improved muscle performance. When applied in the insertion to origin mode, it is claimed to inhibit excessive muscle contractions. Objective: Investigate whether KT applied in the insertion to origin mode could reduce the exaggerated reflex contraction of spastic muscles. Design: Randomized crossover trial, with a restricted block randomization. Setting: Clinical laboratory and swimming pool. Patients: 7 para-swimmers. Intervention: KT, applied in inhibitory mode, to investigate its effect on knee extensor spasticity. Main Outcome Measures: Primary outcome is stretch reflex, as compared to clinical assessment of spasticity by Modified Ashworth Scale (MAS) and self-perceived spasticity by Numeric Rating Scale (NRS). Secondary outcomes were Medical Research Council (MRC) for strength of knee extensor muscles, and chronometric swimming performance in $100 \mathrm{~m}$ freestyle. Results: KT decreased significantly the amplitude of stretch reflex $(p<0.001)$, while the placebo treatment produced no significant effects. Scores of MRC for strength and MAS did not change after KT, whereas NRS scores for spasticity significantly decreased $(p=0.001)$. The swimming performance was significantly improved after KT treatment as compared to baseline $(p<0.01)$. Conclusions: This exploratory study performed on para-athletes suggests that KT could reduce spasticity. This outcome has threefold implications for clinical, rehabilitation and sport methods.

Keywords: muscle spasticity; Kinesio Taping; stretch reflex; athletes; para-athlete; electromyography 


\section{INTRODUCTION}

Muscle tone is the resistance felt by the examiner when he or she manipulates a joint through a range of movement, while the subject attempts to relax. Muscle hypertonia, i.e. the increase of muscle tone, is a common finding in patients with upper motor neuron syndrome (UMNS) following several neurological diseases including cerebral palsy, stroke, multiple sclerosis, spinal cord injury and traumatic brain injury.

In patients affected by UMNS, muscle hypertonia is often related to the presence of spasticity, defined as a disorder of movement and posture, characterised by velocity-dependent increase in the tonic stretch reflexes ("muscle tone") with exaggerated tendon jerks resulting from hyperexcitability of the stretch reflex (Lance, 1980). Compelling evidence exist that spasticity is due to an exaggerated stretch reflex (Marinelli et al., 2017; Trompetto et al., 2019a; Trompetto et al., 2019b).

Spasticity may assist motor function and motor coordination, yet it may also contribute to motor disability, limiting joint range of motion and antagonist muscle strength. This is particularly an issue for para-athletes affected by UMNS, in which spasticity, as well as co-contraction and inexhaustible clonus in fatigued conditions, particularly in lower limbs, may affect performance during competitions (Puce et al., 2018), reduce efficacy of training sessions and have a detrimental effect on functioning in daily activities.

Muscle hypertonia in patients with UMNS can be divided into two components: hypertonia produced by an exaggerated stretch reflex, which corresponds to spasticity, and hypertonia due to muscle fibrosis and muscle shortening (i.e. muscle contracture), often referred as non-reflex hypertonia or intrinsic stiffness. The latter component makes a significant contribution to hypertonia in UMNS patients (Dietz et al., 1983). Both muscle fibrosis and muscle shortening are secondary effects to muscle immobilization in a shortened position (Gracies, 2005) and are therefore likely present in sedentary and untrained patients, but minimally present in highly trained para-athletes. While it is difficult to distinguish reflex and non-reflex hypertonia clinically 
(Thompson et al. 2005), the assessment of the stretch reflex by surface ElectroMyoGraphy (sEMG) can provide a selective measure of spasticity (McGibbon et al., 2013).

Kinesio Taping (KT) is an emerging taping technique (Kase et al., 2013) based on the use of a thin elastic tape mimicking the elastic qualities of the skin. KT has been designed to produce micro convolutions or folds in the skin in the attempt to promote the flow of blood and lymphatic fluids in the underneath tissues. According to its manufacturers, KT produces several clinical effects, including pain relief, edema absorption and improved muscle performance and literature reports either in support of or against these claims can be found (Castro-Sanchez et al., 2012; Ozmen et al., 2016; Wang et al., 2018). When the tape is applied at the origin of the muscle and extended to its insertion using $25-50 \%$ of the available tension (origin to insertion mode), $\mathrm{KT}$ is claimed to facilitate muscle contraction. On the contrary, the application of KT from insertion to origin using $15-25 \%$ of the available tension (insertion to origin mode) is considered to inhibit muscle function (Kase et al., 2013). From a theoretical point of view, the latter mode of KT application could be suitable to reduce the exaggerated stretch reflex contraction of spastic muscles, enabling cutaneous afferent receptor signals that modify the excitability of motor units and modulate the activity of the proprioceptive reflex loops (Yeung \& Yeung, 2016).

To our knowledge, there are only three papers in which KT was used to treat muscle hypertonia (Boeskov et al., 2014; Karadag-Saygi et al., 2010; Tamburella, et al., 2014). In all these studies, muscle hypertonia was assessed clinically, using the Modified Ashworth Scale (MAS) (KaradagSaygi et al., 2010; Tamburella et al., 2014), the passive range of movement (p-ROM) (KaradagSaygi et al., 2010; Tamburella et al., 2014) and the Tardieu Scale (TS) (Boeskov et al., 2014). As clinical evaluation cannot clearly distinguish between reflex and non-reflex hypertonia (Gracies, 2005), it is unknown if KT acts on spasticity or the intrinsic component of muscular hypertonia. Knowing which component of muscle hypertonia KT is acting on would allow to better understand the mechanism of action of KT and to use the treatment more appropriately either in patients with spasticity or in those with intrinsic hypertonia. 
The primary aims of our study are (i) investigating effect of KT applied in the inhibitory mode on the stretch reflex component of spasticity, using sEMG analysis, and (ii) exploring the beneficial or detrimental effects of KT on the swimming performance of para-swimmers. Indeed, while decreased spasticity may improve motor function and motor coordination, it is important to ensure that KT does not affect the athlete's strength, propulsive capability and ability to perform. The recruited population of top-performing para-swimmers is very specific in comparison with the typical population of untrained UMNS patients, in that they are characterized by increased muscle elasticity and reduced fibrosis and pain, thanks to long lasting and constant physical exercise.

\section{METHODS}

\section{Design}

The present study was designed as a randomized crossover trial, using a restricted block randomization to assign to the KT intervention in the first part of the study and the placebo treatment in the second part or vice versa, as sketched in Fig. 1.

None of the participants had ever used any type of KT before the present experiment. Throughout the study period, the participants were instructed to keep both their drug therapy (one of them assumed a drug specific for spasticity) and diet unchanged. Moreover, in this period they were subject to a low intensity aerobic swimming training, with volume, duration and weekly frequency of training tailored to the characteristics of each athlete in such a way that they performed the tests in the same psychophysical conditions.

Primary endpoint of the study was stretch reflex reduction after KT application, as compared to reduction of MAS and NRS (for spasticity).

Secondary endpoints were: no MRC for knee extensor muscle strength changes after KT application and improvement of chronometric swimming performance after KT treatment. 


\section{Participants}

Seven young adult subjects were enrolled for this study among the members of a swimming club recognized by the International Paralympic Committee (named "Nuotatori Genovesi”), according to the following criteria: 1) velocity-dependent hypertonia of quadriceps muscle with increased patellar reflex affecting one or both sides ranging from 1-3 according to the MAS; 2) no pain at the level of the lower limbs, evaluated by the 1-10 Numeric Rating Scale (NRS) for pain; 3) echo intensity of the rectus femoris muscle, as measured by ultrasonography at the enrollment stage (affected/more affected side or right side in the case of symmetrical knee extensor hypertonia), corresponding to grades I-II of the Heckmatt scale (Heckmatt et al., 1982); 4) no use of botulinum toxin in the last 6 months; 5) no pathological conditions interfering with the assessment of the quadriceps stretch reflex; 6) Gross Motor Function Classification System (GMFCS) level between II and III.

The study was carried out in accordance with The Code of Ethics of the World Medical Association (Declaration of Helsinki) for experiments involving humans; a written informed consent was obtained from all participants prior to participation in the study. The project was approved by the institutional review committee.

\section{Procedures}

\section{a) Kinesio Taping Interventions}

In the case of symmetrical distribution of knee extensor spasticity, KT was applied on the right side; in the case of unilateral or asymmetrical distribution, KT was applied on the affected or more affected side. All the participants were taped by the same certified KT physiotherapist using a 5-cm width Kinesio Tex ${ }^{\circledR}$ Gold Finger PrintTM. Before the application of the tape, the skin was cleaned and shaved, and covered with few sweeps of adhesive spray (Tensospray®). During the KT application, the participant was lying close to the edge of a couch, with the treated lower limb hanging aside, as depicted in Fig. 2. This position allowed the hip to be extended and the knee to be 
in full passive flexion, simultaneously, so that when the participant was back in neutral position, microconvolutions were generated in the skin. One "Y" strip was firstly applied above the muscle belly of rectus femoris, with the two tails surrounding the lateral and medial border of patella; then two "I" strips were applied above the muscle bellies of vastus medialis and vastus lateralis. All the three strips were applied in the insertion to origin mode using approximately $20 \%$ of the available tension and were rubbed to maximize their adhesion to the skin. To ensure that the correct tension was applied, the reference length corresponding to $100 \%$ of available tension was measured by a ruler, after a first full stretch of the tape.

\section{b) Placebo treatment}

For the placebo, common adhesive strips similar in shape and color to the Kinesio Tex ${ }^{\circledR}$ Gold Finger PrintTM were used and applied to subjects using the same procedure as for the KT intervention and by the same operator.

\section{c) Clinical assessments of spasticity and strength}

To evaluate knee extensor spasticity, the MAS was used. Furthermore, a 0-10 Numeric Rating Scale (NRS) was used by all participants to measure the level of self-experienced spasticity of the lower limb under test, over a 24-hour period (Farrar et al., 2008). Medical Research Council (MRC) scale for strength was used to quantify knee extensor strength.

\section{d) Stretch reflex assessment}

In order to perform the stretch reflex evaluation, participants were sitting on the edge of a couch, with the back leaning against a support and the legs hanging down. Participants were asked to remain relaxed during the assessment. The examiner moved the participant's leg from the extended position $\left(0^{\circ}\right.$ between the leg and thigh) to the flexed position $\left(90^{\circ}\right.$ between the leg and thigh) and vice versa continuously, until 9 passive sinusoidal movements were collected. In each subject, the 
optimal velocity to evoke a stretch reflex was chosen, taking into account that low values could not be able to elicit the reflex or could elicit a reflex too small to be investigated (especially in subjects with a low degree of spasticity), while high values could produce discomfort to the subject and excessive fatigue in the examiner. The same velocities, reported in Table 2, were maintained at baseline, and after KT and placebo treatments.

EMG activity from the rectus femoris muscle was recorded through surface preamplified electrodes (TSD150B, Biopac Systems Inc, USA) following SENIAM (Surface Electromyography for NonInvasive Assessment of Muscles) guidelines (Hermens, 1999). The signal was acquired by a MP150 unit (Biopac Systems Inc, USA) with a $2 \mathrm{KHz}$ sampling rate and underwent a Blackman -61dB 20$300 \mathrm{~Hz}$ band-pass filter for off line processing (AcqKnowledge 3.8.1 software by Biopac Systems Inc, USA). For the kinematic recording of the passive leg movements, we used a TSD130B twinaxis electronic goniometer (Biopac Systems Inc, USA) connected to the Biopac MP150 data acquisition system. The goniometer was placed across the knee joint in order to optimally record the angle during the joint displacements; a sampling rate of $2 \mathrm{KHz}$ was used.

Onset and termination times of each flexion movements were visually detected on the goniometer trace displayed on the computer screen using a display gain of $20 \% \mathrm{~cm}$ and a temporal window of $340 \mathrm{~ms} / \mathrm{cm}$. Stretch reflex amplitude was measured as the mean amplitude of the rectified EMG (averaged rectified value, ARV) during each one of the 9 flexion movements (from extended position to flexed position).

Stretch reflex and clinical assessment were performed three times, namely at baseline just before the treatment (T0) and 48 hours after the application of the KT or the placebo (T1 or T2).

\section{e) In-water tests}

After the clinical and sEMG evaluations at baseline and KT or placebo treatment, and after an adequate warm-up session, the athletes were asked to perform $100 \mathrm{~m}$ freestyle at maximum speed, in a $50 \mathrm{~m}$ swimming pool. All the in-water test were performed in the same part of the day, namely 
in the morning hours, in order to minimize the effect of circadian cycle on spasticity, and in the first training session of the week, in order to have similar psychophysical conditions for all the participants. The chronometric performance of each athlete was measured by a Italian Paralympic Swimming Federation (FINP) certified technician. Athletes at baseline swam close (within few percent) to their personal best times.

\section{h) Statistical analysis}

A repeated-measures ANOVA with three levels (baseline, KT and placebo) was performed between the sEMG signals averaged over the nine collected passive movements. Significant interactions in the ANOVA were assessed by post hoc Newman-Keuls tests. Similarly, repeated-measures ANOVA tests, followed by post hoc Newman-Keuls tests, were performed to assess statistical significance of the reduction of NRS for spasticity and chronometric times in the $100 \mathrm{~m}$ freestyle tests after KT application and placebo.

Pearson coefficient $r$ was calculated to assess correlations between stretch reflex and NRS scores and between stretch reflex and $100 \mathrm{~m}$ freestyle times in all the data from the different conditions pooled together.

Statistical significance was set at $\mathrm{p}<0.05$.

\section{RESULTS}

All demographic, disease related and athletic variables of the 7 subjects are reported in Table 1 (age $22.0 \pm 2.83$ years; height $164.8 \pm 6.21 \mathrm{~cm}$; weight $64.2 \pm 3.9 \mathrm{~kg}$; GMFCS II-III).

A stretch reflex was evoked in all the 7 enrolled subjects. Table 2 reports the results of EMG and clinical assessment at baseline, 48 hours after KT or placebo. In each subject a significant reduction of the stretch reflex amplitude after KT was found, and no significant change after placebo (see Fig. 3 for a typical acquisition). In Fig. 4a, the mean changes of the stretch reflex amplitude after the KT and placebo conditions are shown. A significant reduction by $36.9 \%$ was found in the KT condition 
with respect to baseline ( $p=0.0009$ ), as well as by $35.4 \%$ in the KT condition with respect to the placebo one $(p=0.0006)$. The scores of MAS and MRC for strength did not change appreciably, either after KT or after placebo, while the scores of NRS for spasticity decreased significantly after KT, as shown in Fig. 4b. A significant reduction by $30.0 \%$ was observed after KT with respect to baseline $(p=0.0008)$ and by $28.2 \%$ after KT compared to placebo $(p=0.0006)$. In Table 3 the $100 \mathrm{~m}$ swimming chronometric performances at baseline, after KT and after placebo treatments are reported for each participant. All performances improved after KT treatment, despite the large spread of chronometric times related to the spread in functional impairment of participants. As shown in Fig. 4c, the mean chronometric time decreased by $-2.7 \%$ after KT treatment with respect to baseline $(p=0.0017)$, whereas placebo treatment did not induce any significant change. Finally, in Fig. 5 the linear regressions of stretch reflex amplitudes and NRS scores (panel a) and of stretch reflex amplitudes and $100 \mathrm{~m}$ freestyle times (panel b) are shown, respectively. In both cases, we find positive and statistically significant correlations between the variables, namely $r=0.89$, $p<0.00001$ for stretch reflex amplitudes and NRS scores and $r=0.58, p=0.006$ for stretch reflex amplitudes and $100 \mathrm{~m}$ freestyle times.

\section{DISCUSSION}

The aim of this study was to investigate the effect of KT, applied in its inhibitory mode, on knee extensor spasticity using stretch reflex as primary outcome measure, in comparison with MAS and NRS for spasticity scores. Secondary outcome measures were MRC of knee extensor muscle strength, and chronometric performances in $100 \mathrm{~m}$ freestyle swimming. We investigated a highly specific group of para-athletes without pain and with an ultrasound muscle picture showing either no muscle fibrosis or only mild muscle fibrosis.

The main result of the present study is that KT decreased significantly the amplitude of stretch reflex, while placebo treatment produced no significant effects. To our knowledge, this is the first study showing the effect of KT on the stretch reflex in UMNS subjects. In addition, while MAS 
scores did not change after KT, NRS scores for spasticity decreased significantly. Our result of decreased amplitude of stretch reflex after KT is particularly relevant for para-athletes. Indeed, despite the use of KT is not allowed during competitions, the inhibition of the stretch reflex may be highly beneficial in training sessions, allowing the athletes to sustain prolonged or intensive training sessions with limited fatigue-induced amplification of positive signs of the UNMS such as spasticity, co-contraction and muscle spasms, which detrimentally interfere with the swimming technique and performance (Puce et al., 2018).

We found no changes in scores of MRC for strength after KT. The absence of weakness induced by $\mathrm{KT}$ is also an important finding. In fact, most treatments that reduce spasticity have the side effect of reducing strength. This occurs not only in the case of botulinum toxin, considered the therapeutic gold standard for spasticity, but also in the case of drugs used to treat spasticity, from baclofen to benzodiazepines. It often happens that the concomitant reduction in muscle strength limits the effectiveness of these treatments and consequently their clinical applicability. This result is again particularly relevant for para-athletes. Indeed, in principle we could have expected that the KT applied in the inhibitory mode induced weakness and hindered the muscle functionalities in swimming, which could counteract and ultimately neutralize the beneficial effect of decreased spasticity. On the contrary, our results show that the application of KT yields a clear and statistically significant improvement of the swimming chronometric performance.

Our regression analysis, indicating positive strong correlations between stretch reflex amplitudes and NRS scores and between stretch reflex amplitudes and $100 \mathrm{~m}$ freestyle times suggest that sEMG, NRS and performance assessment could be complementary and consistent methods to measure spasticity.

Spasticity can be the direct cause of pain (Truini et al., 2013) and in turn pain may increase spasticity, creating a progressive rise of pain and disability (Ward et al., 2002). Previous studies state that $\mathrm{KT}$ is an effective treatment in relieving pain in patients with orthopedic and neurological diseases (Castro-Sanchez et al., 2012; Kase et al., 2013). Both neurological and musculoskeletal 
mechanisms were proposed to account for pain relief, for example, KT could provide pain inhibitory afferent stimuli that interfere with the transmission of mechanical and painful stimuli (Paolini et al., 2011), or else it could stimulate Golgi receptors to transmit information to the central nervous system to inhibit hypertonic muscle activity responsible for pain (Kase et al., 1996). Therefore, it may be argued that KT has an indirect effect on spasticity through its action on pain. Instead, the present findings, obtained in subjects without chronic pain, suggest a direct effect of KT on spasticity, i.e. not mediated by pain reduction.

The effects of KT treatment applied in the inhibitory mode on muscle hypertonia were investigated in three previous works (Boeskov et al., 2014; Karadag-Saygi et al., 2010; Tamburella et al., 2014). Karadag-Saygi et al. investigated the effect of KT treatment in stroke patients with hypertonia of plantar flexors, receiving botulinum toxin type A (BoNT-A). Among the outcome measures, namely MAS, passive ankle dorsiflexion (p-ROM) and gait parameters, only p-ROM resulted significantly more reduced in the KT group as compared to the placebo group (Karadag-Saygi et al., 2010).

Tamburella et al. showed that KT reduced Visual Analogue Scale (VAS) for spasticity of plantar flexors muscles in 11 patients affected by incomplete spinal cord injury. MAS score was not significantly decreased after KT. A significant effect of KT on ankle p-ROM, clonus, pain and several kinematic gait and EMG data was also reported. A decrease of co-contraction activity of plantar flexors by KT treatment during a foot dorsal flexion motor task was also observed. All these effects were discussed in terms of sensory modulation produced by KT treatment (Tamburella et al., 2014).

Boeskov et al. investigated walking function and hypertonia of knee extensors, assessed by Tardieu scale, before and after application of KT to the anterior thigh and knee in 32 stroke patients. The results showed an immediate improvement of walking function. Only a trend indicating a lesser degree of muscle hypertonia was observed. Again, the results were discussed in terms of modulation of proprioceptive inputs induced by KT treatment (Boeskov et al., 2014). 
The results of our study confirm and expand the findings from the above mentioned works, confirming that KT can reduce muscle hypertonia without producing any strength reduction. Furthermore, our results confirm that subjective measures (VAS and NRS) are more sensitive than objective clinical scales in capturing changes of muscle hypertonia. It must be said, however, that this high sensitivity of NRS and VAS scales is balanced by an equally poor specificity providing a subjective estimation of several positive UMNS signs beside spasticity (including spastic dystonia, co-contraction and muscle spasms), often contaminated also by the negative UMNS signs (weakness and loss of dexterity) (Marinelli et al., 2016). For this reason, in the present work, NRS scale was associated with the most specific measure for spasticity, namely the stretch reflex. In two of the above mentioned studies investigating KT treatment applied in the inhibitory mode in patients with muscle hypertonia, the effect was documented by outcome measure reflecting both the reflex and the intrinsic components of muscle hypertonia, namely p-ROM (Karadag-Saygi et al., 2010) and MAS (Tamburella et al., 2014). In the only study in which a specific measure for spasticity was used, namely the Tardieu scale, KT treatment showed no significant changes (Boeskov et al., 2014). Therefore, our study is the first to highlight a specific effect of KT on the reflex component of muscle hypertonia, i.e. spasticity. This finding supports the view that the action of KT on muscle hypertonia is due to modulation of peripheral input from muscle and skin to spinal cord (Boeskov et al., 2014; Tamburella et al., 2014).

Our findings suggest that stretch reflex assessment can provide an objective tool to measure spasticity changes induced by KT. We think that the integration of stretch reflex data with patient self-report measures could provide a sensitive and specific picture of spasticity, able to reveal small changes, that could be nevertheless clinically significant.

The main limitations of this study are related to the small sample of subjects, related to the objective difficulty in recruiting many top-performing para-athletes affected by UMNS. An additional limitation is that the chronometric swimming performance may be a poorly sensitive indicator of 
the limb functionality, because para-swimmers often compensate for underuse of their most affected limbs by developing an adapted swimming technique that relies mostly on their non-affected or less affected limbs.

\section{CONCLUSION}

This exploratory study performed on para-athletes suggests that KT could reduce spasticity. Our results have threefold implications for clinical, rehabilitation and sport methods. Firstly, the study suggests that the use of both clinical and neurophysiological measures can provide a selective and sensitive assessment of spasticity. Secondly, our results indicate that the beneficial effects of KT on spasticity may be considered in the design of rehabilitation protocols. Thirdly, if our results on a small sample size are confirmed on a more general population of swimmers affected by spasticity related to damages of central nervous system, KT may be effectively employed in the physical training protocols.

\section{ACKNOWLEDGMENTS}

We wish to thank the para-athletes involved in the study for their kind collaboration.

\section{REFERENCES}

Boeskov B, Carver LT, von Essen-Leise A, Henriksen M. Kinesthetic taping improves walking function in patients with stroke: a pilot cohort study. Topics in Stroke Rehabilitation 2014;21(6):495-501. https://doi.org/10.1310/tsr2106-495

Castro-Sanchez AM, Lara-Palomo IC, Mataran-Penarrocha GA, Fernandez-Sanchez M, SanchezLabraca N, Arroyo-Morales M. Kinesio Taping reduces disability and pain slightly in chronic non-specific low back pain: a randomised trial. Journal of Physiotherapy. 2012;58(2):89-95. https://doi.org/10.1016/S1836-9553(12)70088-7

Dietz V, Berger W. Normal and impaired regulation of muscle stiffness in gait: a new hypothesis 
about muscle hypertonia. Experimental Neurology. 1983;79(3):680-687.

\section{https://doi.org/10.1016/0014-4886(83)90032-8}

Farrar J T, Troxel AB., Stott C, Duncombe P, Jensen MP. Validity, reliability, and clinical importance of change in a 0-10 numeric rating scale measure of spasticity: a post hoc analysis of a randomized, double-blind, placebo-controlled trial. Clinical Therapeutics. 2008;30(5):974-985. https://doi.org/10.1016/j.clinthera.2008.05.011

Gracies JM. Pathophysiology of spastic paresis. I: Paresis and soft tissue changes. Muscle \& Nerve. 2005;31(5):535-551. https://doi.org/10.1002/mus.20284

Heckmatt JZ., Leeman S, Dubowitz V. Ultrasound imaging in the diagnosis of muscle disease. The Journal of Pediatrics. 1982;101(5):656-660. https://doi.org/10.1016/s0022-3476(82)80286-2

Hermens HJ. European recommendations for surface ElectroMyoGraphy: results of the SENIAM project. (H. Hermens, Ed.). Roessingh Research and Development BV, 1999.

Karadag-Saygi E, Cubukcu-Aydoseli K, Kablan N, Ofluoglu D. The role of kinesiotaping combined with botulinum toxin to reduce plantar flexors spasticity after stroke. Topics in Stroke Rehabilitation. 2010;17(4):318-322. https://doi.org/10.1310/tsr1704-318

Kase K, Tatsuyuki H, Tomoko O, Development of kinesio tape. Kinesio taping perfect manual. Kinesio Taping Association, 6(10), pp. 117-118, 1996.

Kase K, Wallis J, Kase T. Clinical Therapeutic Applications of the Kinesio Taping Method. Albuquerque, NM: Kinesio Taping Association International, 2013.

Lance JW. Symposium synopsis. In R. G. Feldman, R. R. Young, \& K. P. Koella (Eds.), Spasticity: Disorder of Motor Control (pp. 485-494). Chicago, 1980.

Marinelli L, Mori L, Canneva S, Colombano F, Curra A, Fattapposta F, Trompetto C. The effect of cannabinoids on the stretch reflex in multiple sclerosis spasticity. International Clinical Psychopharmacology. 2016;31(4):232-239. https://doi.org/10.1097/YIC.0000000000000126

Marinelli L, Currà A, Trompetto C, Capello E, Serrati C, Fattapposta F, Pelosin E, Phadke C, Bandini F. Spasticity and spastic dystonia: the two faces of velocity-dependent hypertonia. $J$ 
Electromyogr Kinesiol. 2017;37:84-89.

McGibbon CA., Sexton A, Jones M, O’Connell C. Elbow spasticity during passive stretch-reflex: clinical evaluation using a wearable sensor system. Journal of Neuroengineering and Rehabilitation. 2013;10(1):61. https://doi.org/10.1186/1743-0003-10-61

Ozmen T, Aydogmus M, Dogan H, Acar D, Zoroglu T, Willems M. The effect of Kinesio Taping on muscle pain, sprint performance, and flexibility in recovery from squat exercise in young adult women. J Sport Rehabil. 2016;25(1):7-12. https://doi.org/10-1123/jsr.2014-0243

Paolini M, Bernetti A, Fratocchi G, Mangone M, Parrinello L, Cooper MP. Kinesio Taping applied to lumbar muscles influences clinical and electromyographic characteristics in chronic low back pain patients. Eur. J. Phys. Rehab. Med. 2011;47:237-244

Puce L, Marinelli L, Pierantozzi E, Mori L, Pallecchi I, Trompetto C. Training methods and analysis of races of a top level Paralympic swimming athlete. J Exerc Rehabil. 2018;14(4):612-620. https://doi.org/10.12965/jer.1836254.127

Tamburella F, Scivoletto G, Molinari M. Somatosensory inputs by application of KinesioTaping: effects on spasticity, balance, and gait in chronic spinal cord injury. Frontiers in Human Neuroscience. 2014;8:367. https://doi.org/10.3389/fnhum.2014.00367

Thompson AJ, Jarrett L, Lockley L, Marsden J, Stevenson VL. Clinical management of spasticity. Journal of Neurology, Neurosurgery, and Psychiatry. England. 2005. https://doi.org/10.1136/jnnp.2004.035972

Trompetto C, Curra A, Puce L, Mori L, Serrati C, Fattapposta F, Marinelli L. Spastic dystonia in stroke subjects: prevalence and features of the neglected phenomenon of the upper motor neuron syndrome. Clinical Neurophysiology: Official Journal of the International Federation of Clinical Neurophysiology. 2019a;130(4):521-527.

https://doi.org/10.1016/j.clinph.2019.01.012

Trompetto C, Marinelli L, Puce L, Mori L, Serrati C, Fattapposta F, Curra A. "Spastic dystonia” or "Inability to voluntary silence EMG activity"? Time for clarifying the nomenclature. Clinical 
Neurophysiology: Official Journal of the International Federation of Clinical

Neurophysiology. Netherlands. 2019b. https://doi.org/10.1016/j.clinph.2019.03.009

Truini A, Barbanti P, Pozzilli C, Cruccu G. A mechanism-based classification of pain in multiple sclerosis. Journal of Neurology. 2013;260(2):351-367. https://doi.org/10.1007/s00415-012$\underline{6579-2}$

Wang Y, Gu, Chen J, Luo W, He W, Han Z, Tian J. Kinesio taping is superior to other taping methods in ankle functional performance improvement: a systematic review and meta-analysis. Clin Rehabil. 2018;32(11):1472-1481. https://doi.org/10.1177/0269215518780443

Ward AB, Kadies M. The management of pain in spasticity. Disability and Rehabilitation. 2002;24(8):443-453. https://doi.org/10.1080/09638280110108878

Yeung SS, Yeung EW. Acute Effects of Kinesio Taping on Knee Extensor Peak Torque and Stretch Reflex in Healthy Adults. Medicine. 2016;95(4):e2615.

https://doi.org/10.1097/MD.0000000000002615 


\section{Table captions}

Table 1: Demographic, disease-related and athletic variables of the 7 subjects included in the study. UMNS: Upper Motor Neuron Syndrome; GMFCS: Gross Motor Function Classification System; TS: Training Session.

Table 2: Results of EMG and clinical assessment at baseline, after KT treatment and after placebo treatment. ARV = Average Rectified Value of the EMG signal relative of the stretch reflex amplitude; $\mu \mathrm{V}=$ micro-Volts; MAS: Modified Ashworth Scale; NRS: numeric rating scale for muscle stiffness.

Table 3: Chronometric results of $100 \mathrm{~m}$ swimming performances at baseline, after KT treatment and after placebo treatment. Percent variations with respect to baseline are also indicated. 


\section{Figure captions:}

Figure 1: Sketch of randomized cross-over assignment and study design. Dotted and continuous lines in participants' silhouettes indicate affected and non-affected limbs, respectively. The timeline of the study is the following: 1) ultrasonography analysis; 2) after one week, assessment of primary and secondary outcomes at baseline (T0); 3) application of KT/placebo treatment; 4) after 48h, assessment of primary and secondary outcomes at first intervention (T1); 5) after one week without tape treatment, application of placebo/KT treatment; 6) after $48 \mathrm{~h}$, assessment of primary and secondary outcomes at second intervention (T2).

Figure 2: Sketches of the application points of the KT or placebo (left) and of the patient position during application (right).

Figure 3: Typical acquisitions of the stretch reflex EMG signal at baseline, after the application of the KT treatment and after the application of the placebo treatment (Subject 6).

Figure 4: Mean values and standard deviations of the stretch reflex amplitude (a), NRS score for spasticity (b) and $100 \mathrm{~m}$ freestyle chronometric performances of the 7 participants at baseline and after the KT and placebo applications. ${ }^{*} \mathrm{p}<0.01, * * \mathrm{p}<0.001$.

Figure 5: Linear regression between the EMG amplitudes and NRS scores for spasticity (panel a) and between EMG amplitudes and 100 m freestyle times (panel b). 
Table 1

\begin{tabular}{cccccccc}
\hline $\begin{array}{c}\text { Subject } \\
\text { number }\end{array}$ & Age & Sex & $\begin{array}{c}\text { Topography of } \\
\text { UMNS signs }\end{array}$ & $\begin{array}{c}\text { UMNS } \\
\text { etiology }\end{array}$ & GMFCS & $\begin{array}{c}\text { TS per week } \\
\text { (number) }\end{array}$ & $\begin{array}{c}\text { Single TS } \\
\text { duration } \\
\text { (hours) }\end{array}$ \\
\hline 1 & 19 & M & Spastic tetraparesis & Cerebral palsy & III & 3 & 1 \\
2 & 23 & M & Spastic paraparesis & Cerebral palsy & II & 6 & 2 \\
3 & 21 & M & Spastic tetraparesis & Cerebral palsy & III & 3 & 1 \\
4 & 23 & F & Spastic hemiparesis & Cerebral palsy & II & 6 & 2 \\
5 & 26 & M & Spastic hemiparesis & Cerebral palsy & II & 6 & 2 \\
6 & 24 & F & Spastic paraparesis & Cerebral palsy & II & 5 & 2 \\
7 & 18 & M & Spastic tetraparesis & Cerebral palsy & III & 3 & 1 \\
\hline
\end{tabular}


Table 2

Subject Velocity Stretch reflex amplitude mean $(\mu \mathrm{V})$ number to evoke baseline KT placebo \begin{tabular}{cc}
\multicolumn{2}{c}{ MAS } \\
\hline baseline
\end{tabular} NRS for spasticity $\mathrm{SR}(\%)$

\begin{tabular}{ccccccccccc}
\hline 1 & 45 & 15.36 & 10.63 & 15.06 & 3 & 3 & 3 & 7 & 4 & 7 \\
2 & 45 & 9.28 & 5.94 & 9.84 & 1.5 & 1.5 & 1.5 & 4 & 3 & 4 \\
3 & 45 & 12.48 & 9.24 & 11.5 & 2 & 2 & 2 & 6 & 4 & 6 \\
4 & 90 & 5.33 & 3.86 & 5.29 & 1 & 1 & 1 & 4 & 4 & 4 \\
5 & 90 & 7.59 & 5.62 & 7.93 & 1 & 1 & 1 & 5 & 4 & 5 \\
6 & 90 & 12.06 & 7.44 & 11.94 & 2 & 2 & 2 & 6 & 4 & 6 \\
7 & 45 & 17.52 & 7.55 & 16.31 & 3 & 3 & 3 & 8 & 5 & 7 \\
\hline mean & 64.29 & 11.37 & 7.18 & 11.12 & 1.93 & 1.93 & 1.93 & 5.71 & 4 & 5.57 \\
st. dev. & 24.05 & 4.29 & 2.86 & 3.85 & 0.84 & 0.84 & 0.84 & 1.50 & 0.58 & 1.27 \\
\hline
\end{tabular}


Table 3

\begin{tabular}{|c|c|c|c|c|c|}
\hline \multirow[b]{2}{*}{$\begin{array}{l}\text { Subject } \\
\text { number }\end{array}$} & \multicolumn{3}{|c|}{ Time (s) } & \multicolumn{2}{|c|}{ Time variation $(\%)$} \\
\hline & baseline & KT & placebo & $\begin{array}{l}\text { KT with } \\
\text { respect to } \\
\text { baseline }\end{array}$ & $\begin{array}{l}\text { placebo with } \\
\text { respect to } \\
\text { baseline }\end{array}$ \\
\hline 1 & 175.89 & 167.32 & 175.06 & -4.9 & -0.5 \\
\hline 2 & 71.84 & 69.77 & 72.81 & -2.9 & +1.4 \\
\hline 3 & 215.43 & 211.34 & 215.97 & -1.9 & +0.3 \\
\hline 4 & 84.15 & 82.21 & 83.91 & -2.3 & -0.3 \\
\hline 5 & 103.12 & 102.80 & 104.13 & -0.3 & +1.0 \\
\hline 6 & 106.6 & 103.30 & 105.94 & -3.1 & -0.6 \\
\hline 7 & 152.32 & 147.71 & 151.31 & -3.0 & -0.7 \\
\hline mean & 129.91 & 126.35 & 129.88 & -2.74 & -0.02 \\
\hline st. dev. & 52.69 & 50.98 & 52.47 & & \\
\hline
\end{tabular}




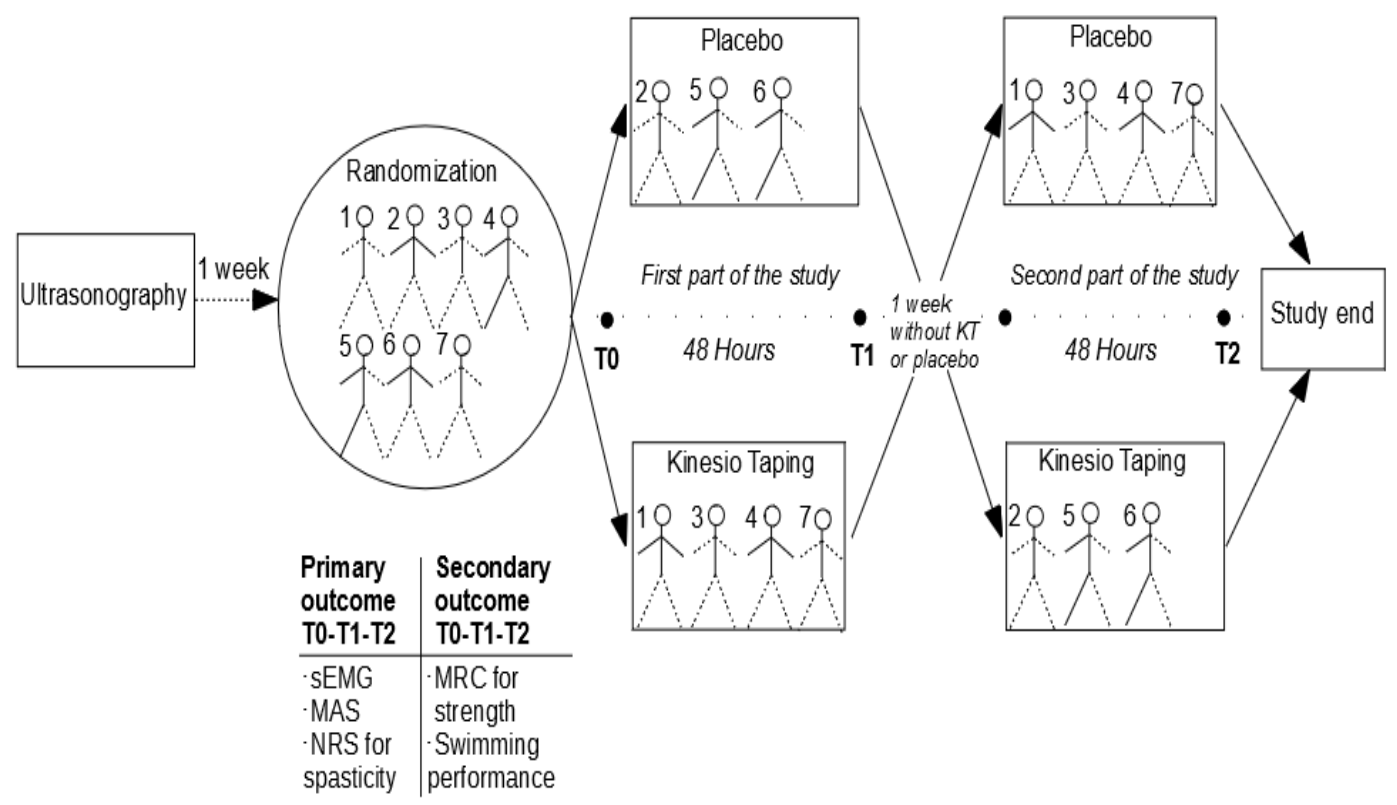

Figure 1 

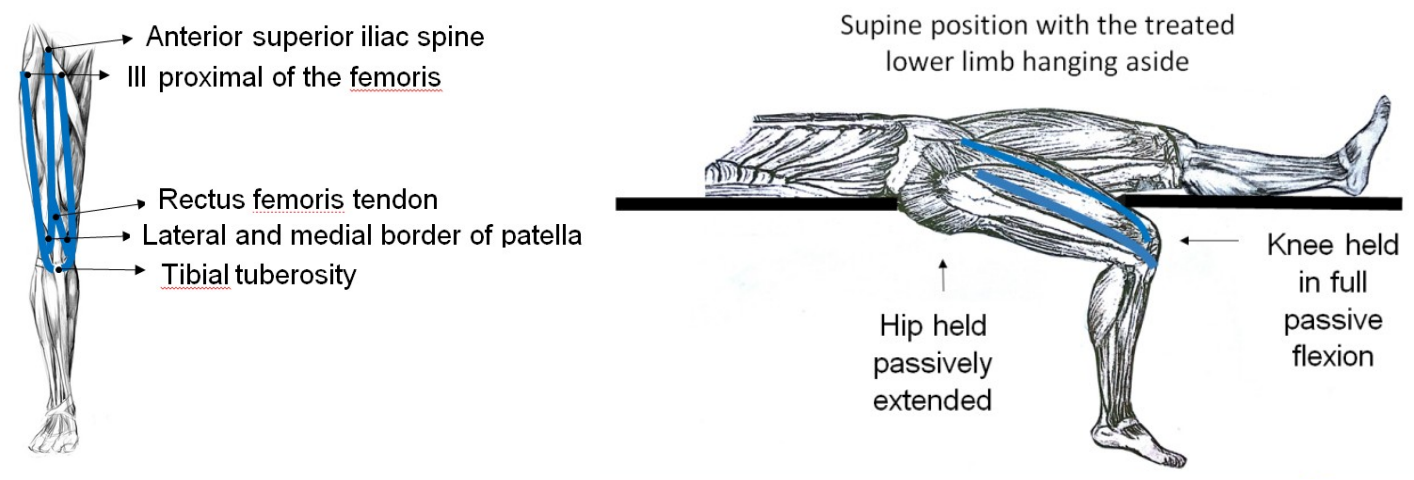

Figure 2 


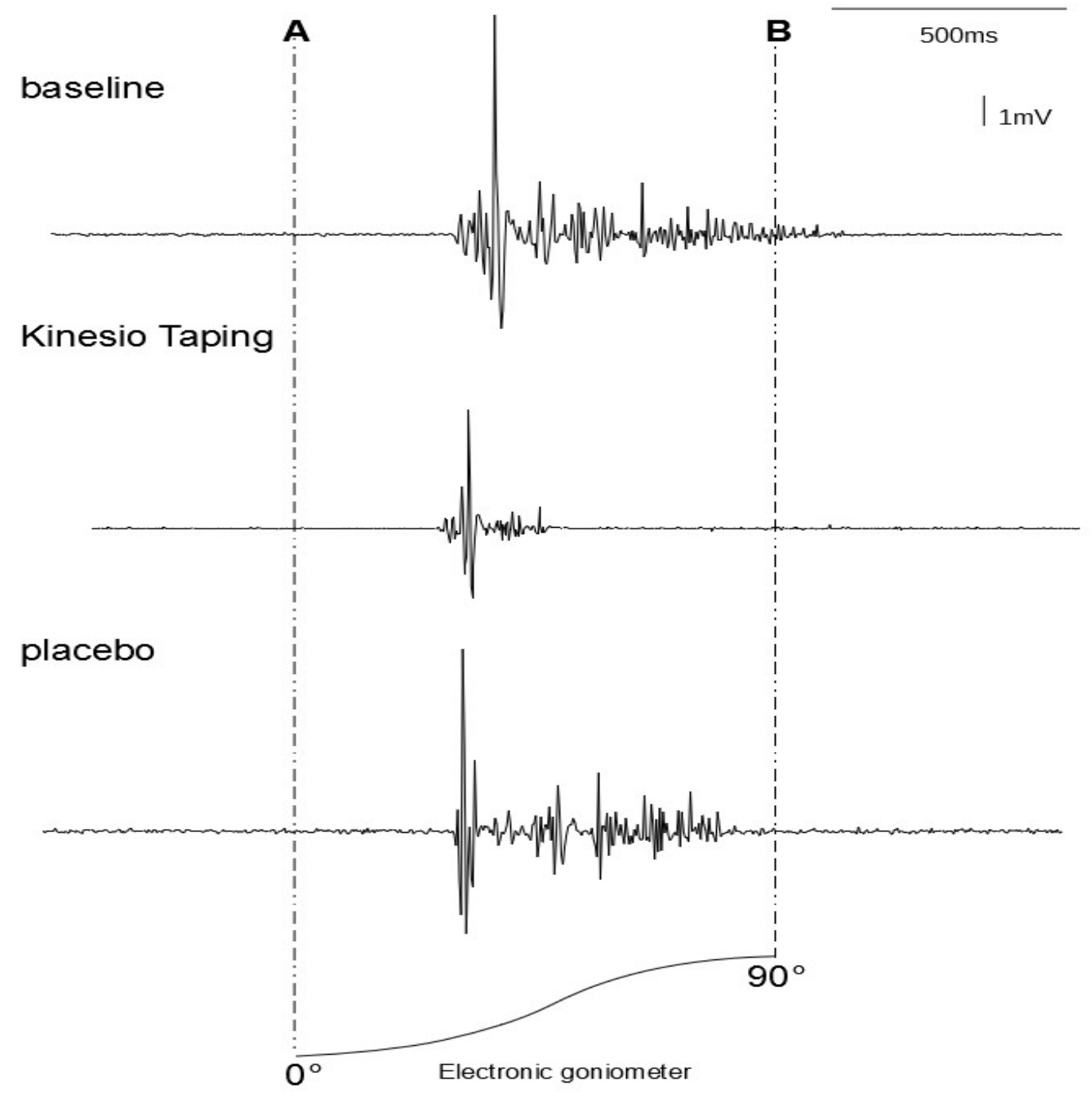

Figure 3 

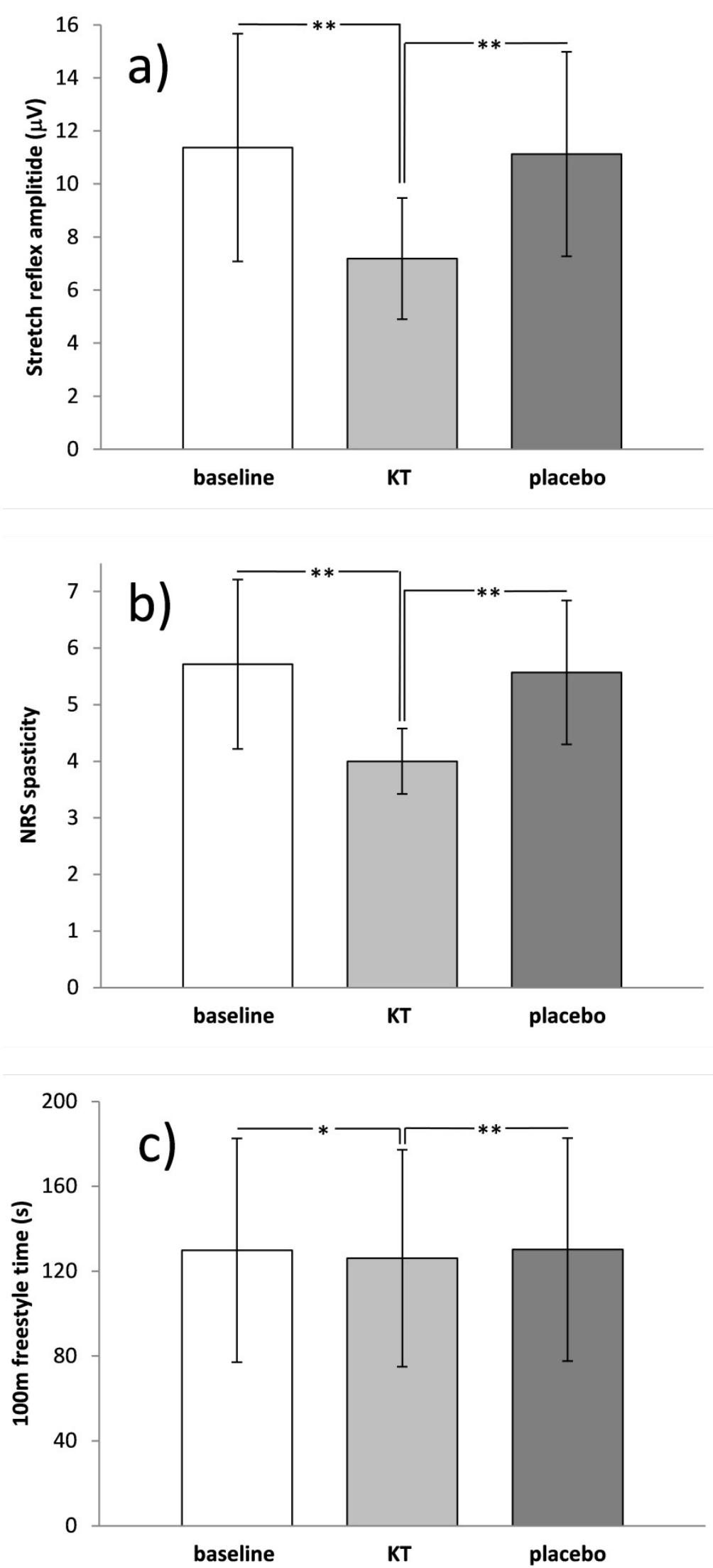

Figure 4 

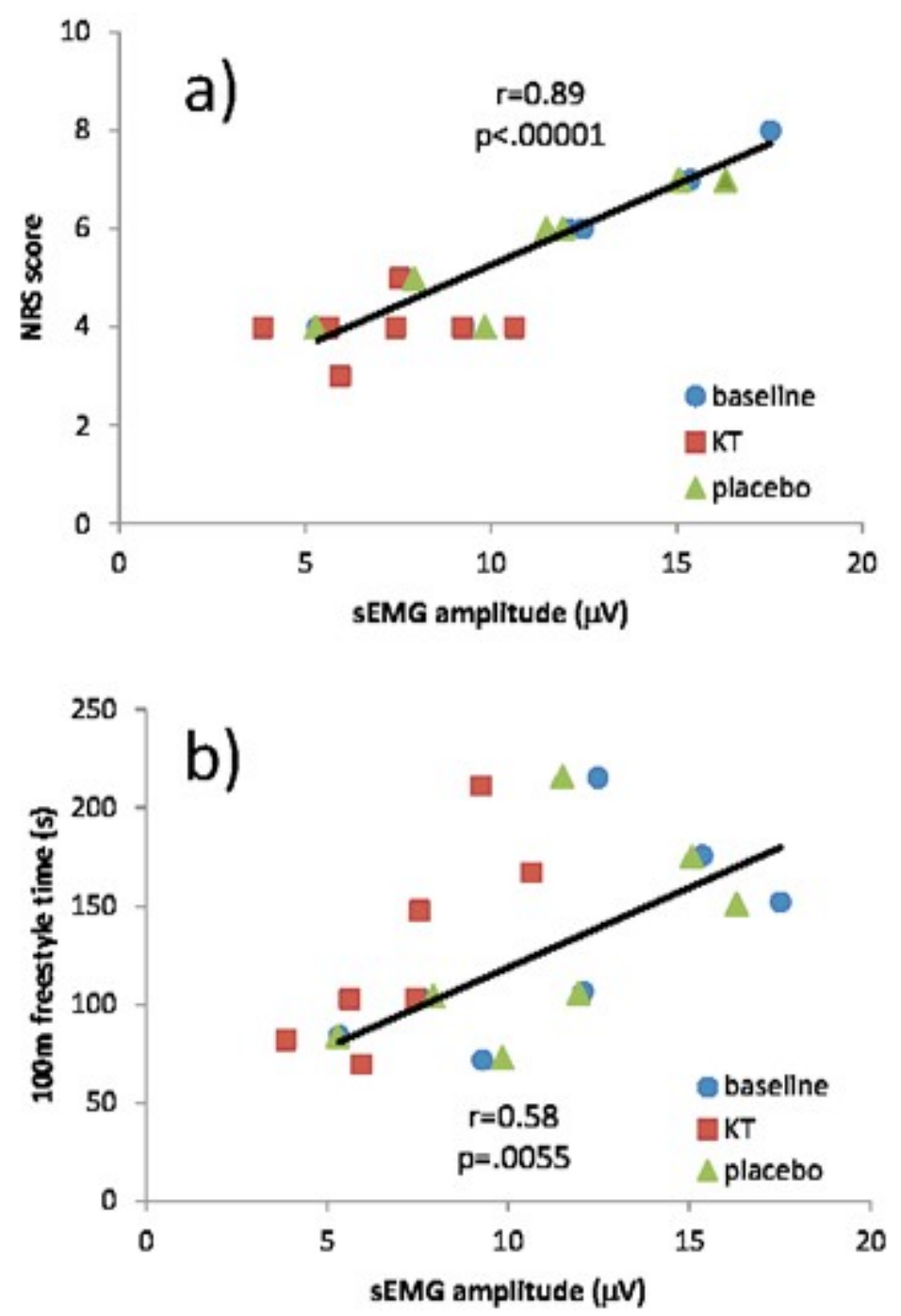

Figure 5 